TITLE:

\title{
Dynamics of deuterated polystyrene-protonated butadiene diblock copolymer micelles by neutron spin echo
}

\section{AUTHOR(S):}

Kanaya, T; Monkenbusch, M; Watanabe, H; Nagao, M; Richter, D

\section{CITATION:}

Kanaya, T ...[et al]. Dynamics of deuterated polystyrene-protonated butadiene diblock copolymer micelles by neutron spin echo. JOURNAL OF CHEMICAL PHYSICS 2005, 122(14): 144905.

\section{ISSUE DATE:}

2005-04-08

URL:

http://hdl.handle.net/2433/39763

\section{RIGHT:}

Copyright 2005 American Institute of Physics. This article may be downloaded for personal use only. Any other use requires prior permission of the author and the American Institute of Physics. 


\title{
Dynamics of deuterated polystyrene-protonated butadiene diblock copolymer micelles by neutron spin echo
}

\author{
T. Kanaya ${ }^{\text {a) }}$ \\ Institute for Chemical Research, Kyoto University, Uji, Kyoto-fu 611-0011, Japan \\ M. Monkenbusch \\ Institut für Festkörperforschung, Forschungszentrum, D-52425 Jülich, Germany \\ H. Watanabe \\ Institute for Chemical Research, Kyoto University, Uji, Kyoto-fu 611-0011, Japan \\ M. Nagao \\ Institute for Solid State Physics, The University of Tokyo, 106-1 Shirakata, Tokai, \\ Ibaraki-ken 319-1106, Japan \\ D. Richter \\ Institut für Festkörperforschung, Forschungszentrum, D-52425 Jülich, Germany
}

(Received 18 October 2004; accepted 1 February 2005; published online 13 April 2005)

\begin{abstract}
We report on neutron spin-echo (NSE) measurements on deuterated styrene-protonated butadiene diblock copolymer micelles in deuterated $n$-decane to investigate the dynamics of butadiene blocks in the corona. Before the NSE measurements, we performed small-angle neutron-scattering (SANS) measurements on the micelles to evaluate the structure to give a basis for the discussion of the dynamics. In the SANS study, we have estimated the form factor $P(Q)$ in terms of a hard-core-shell model from the direct evaluation without curve-fitting procedure while a more flexible core-shell model with the structure factor $S(Q)$ gives a better fit to the observed data. The observed normalized intermediate scattering function $I(Q, t) / I(Q, 0)$ by NSE does not show the collective motions corresponding to the so-called breathing mode but rather single chain motion (Zimm modes) for both the 2 and $20 \mathrm{wt} \%$ micelle solutions. The Zimm decay rate $\Gamma_{z}$ in the micelle solution is slow compared with that in the homopolymer solution. This slowing down is assigned to the effective high concentration in the corona. The differences in $\Gamma_{z}$ between concentrated solutions and the $20 \%$ micellar solution are attributed to end-tethering effect of the corona chains on the core surface. The possible reasons why the breathing mode was not observed in the present micelle system are discussed on the basis of chain density in the corona. () 2005 American Institute of Physics.
\end{abstract}

[DOI: $10.1063 / 1.1879892]$

\section{INTRODUCTION}

When diblock or triblock copolymers are added to a selective solvent, they aggregate into spherical micelles consisting of an inner core of insoluble blocks and an outer corona of soluble blocks, similar to micellization of surfactants. These polymer micelles show interesting properties such as surface activity and absorption of small molecules in the corona, and have high importance in industrial applications. This type of micellization of block copolymers was first reported by Krause ${ }^{1}$ for methyl methacrylate-styrenemethyl methacrylate triblock copolymer in solutions. After the work, extensive studies have been performed on polymer micelles to reveal the structure, thermodynamic, and surface properties and so on. ${ }^{2-4}$ Scattering techniques such as light scattering (LS), ${ }^{5,6}$ small-angle x-ray scattering (SAXS), ${ }^{7,8}$ and small-angle neutron scattering ${ }^{9}$ (SANS) are very powerful tools for the structure studies. Due to relatively low molecular masses of copolymers used in micellar studies

\footnotetext{
${ }^{a)}$ Author to whom correspondence should be addressed. Fax: +81-(0)77438-3146. Electronic mail: kanaya@scl.kyoto-u.ac.jp
}

(mostly up to $2 \times 10^{5} \mathrm{~g} / \mathrm{mol}$ ), LS (Refs. 10-12) with the corresponding low values of scattering vector provides molar mass, aggregation number, and average volume fraction of polymer segments in a micelle, but gives little information about the inner structure. On the other hand, SAXS (Ref. 13) and SANS (Refs. 14-17) with larger values of the scattering vector were shown to be more suitable for studies of the inner structure. In particular, SANS is very powerful because of the possibility of selective variation of the scattering contrast of either part of the copolymer or solvent by the degree of deuteration.

In contrast with extensive studies of the micelle structure, there are few reports on the dynamics of the polymer micelles. Polymer blocks on the core surface that extend into the solvent correspond to tethered chains on a spherical surface. If the grafting density on the surface is high enough to form a dense brush layer, the collective dynamics of the chains driven by the osmotic compressibility and the chain entropic force is expected, which is the so-called breathing mode. This type of collective dynamics was theoretically predicted by de Gennes ${ }^{18}$ for grafting chain brushes on a flat 


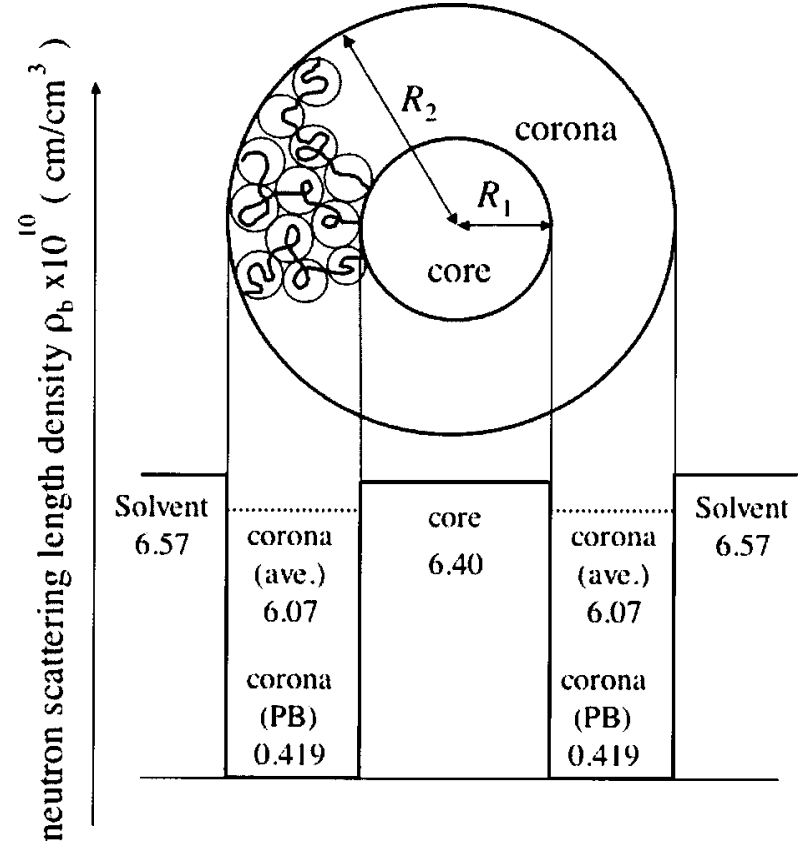

FIG. 1. Schematic drawing of hard-core-shell model with fluctuations of chains in corona and the neutron-scattering length density $\rho_{b}$. The dashed line is an average neutron-scattering density in corona with $\phi_{B}=0.081$.

surface and experimentally probed for spherical micelles ${ }^{19,20}$ and for planar brushes of polyethylene (PE)-polyethylenepropyrene (PEP) platelets ${ }^{21}$ using neutron spin-echo (NSE) technique. This technique can access dynamics in a length scale of a few tens to hundreds of angstroms, ${ }^{22,23}$ and hence is very powerful to study the inner dynamics of polymer micelles. Recently, Matsuoka et al. ${ }^{16}$ have investigated dynamics of polymer micelles in aqueous solutions to find two diffusive modes, the center-of-mass diffusion and a fast mode of still unknown origin.

In this study we have investigated dynamics of micelles of deuterated styrene-protonated butadiene diblock copolymers (PSD-PB) in deuterated $n$-decane, focusing on the chain dynamics in the corona using NSE. Before the dynamics study we have evaluated the structural parameters of the micelle using SANS to give a basis for the discussion of the NSE data.

\section{EXPERIMENT}

The sample used for this experiment is a diblock copolymer consisting of a deuterated polystyrene (PSD) block and a protonated polybutadiene (PB) block, which was prepared by an anionic polymerization method. ${ }^{24}$ The molecular weights of the PSD and PB blocks are 11100 and 23000 , respectively, and the polydispersity $M_{w} / M_{n}$ of the two blocks is less than 1.07. The solvent was deuterated $n$-decane, which is poor for PSD but good for PB, and hence the core and corona of the polymer micelle consist of PSD and PB chains, respectively. We also used $\mathrm{PB}$ homopolymer with $M_{w}$ =34000 and $M_{w} / M_{n}=1.06$ for neutron-scattering experiment. The polymer was also synthesized by an anionic polymerization method. ${ }^{24}$

The neutron-scattering length density of the present system is shown in Fig. 1, together with a schematic drawing of

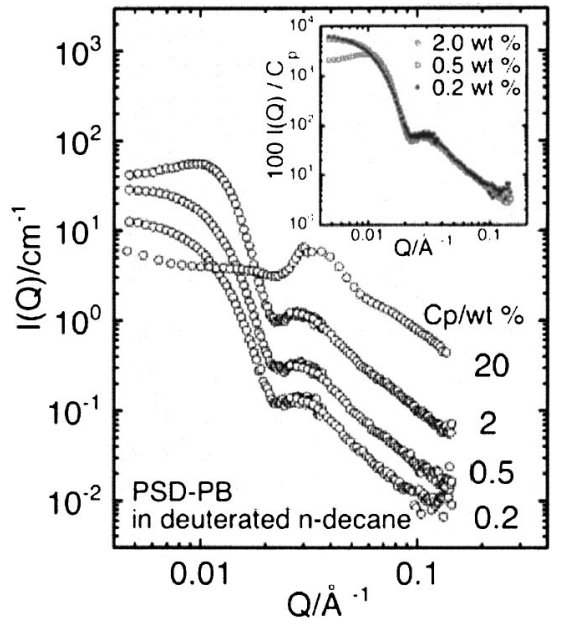

FIG. 2. SANS intensities $I(Q)$ form PSD-PB diblock copolymer micelle in deuterated $n$-decane solutions in absolute unit. Inset shows the normalized intensities to the polymer concentration.

a micelle. The scattering length density $\rho_{b}$ of the PSD core is $6.40 \times 10^{10} \mathrm{~cm} / \mathrm{cm}^{3}$ assuming the density is the same as that in bulk, which almost matches to that of deuterated $n$-decane $\left(6.57 \times 10^{10} \mathrm{~cm} / \mathrm{cm}^{3}\right)$. On the other hand, the scattering length density $\rho_{b}$ of the bulk corona material PB is 0.419 $\times 10^{10} \mathrm{~cm} / \mathrm{cm}^{3}$ while PB chains in the corona are swollen and only occupy a volume fraction $\phi_{B}$ of several percent. The effective scattering length of the shell then is $\phi_{B} \rho_{b, \mathrm{~PB}}$ $+\left(1-\phi_{B}\right) \rho_{b, \text { sol }}$. Since in the present measurements the solvent scattering length density $\rho_{b, \text { sol }}$ is very close to that of the core we mainly observe the PB chains in the corona.

SANS measurements were done using SANS instrument ${ }^{25}$ at the FRJ-2 reactor at the FZ-Jülich, and the SANS-U instrument in JRR-3M reactor, Tokai. ${ }^{26}$ The range of length of scattering vector $Q$ covered by the former and the latter is $5 \times 10^{-3}-0.15 \AA^{-1}$ in this experiment. NSE measurements were carried out using the NSE spectrometer ${ }^{27}$ at the FRJ-2 reactor at the FZ-Jülich. In the NSE measurements the normalized intermediate scattering function $I(Q, t) / I(Q, 0)$ is obtained in the Fourier time range of $0.1-22 \mathrm{~ns}$ and in the $Q$ range of $0.05-0.24 \AA^{-1}$. The NSE measurements were carried out at $40^{\circ} \mathrm{C}$ in order to reduce the solvent viscosity without, however, destroying the micellar structure, the SANS experiments were mainly done at $25^{\circ} \mathrm{C}$ and a few at $40{ }^{\circ} \mathrm{C}$ for comparison. They do not yield significantly different scattering intensities at both temperatures.

\section{RESULTS AND DISCUSSION}

\section{A. Structure of micelles}

Small-angle neutron-scattering measurements were performed on the micelle solutions with concentrations of 0.2 , $0.5,2.0$, and $20 \mathrm{wt} \%$. The observed scattering cross sections $I(Q)$ are plotted in Fig. 2 in absolute units, and the intensities normalized to the concentration $I(Q) / C_{p}$ are also shown in the inset of Fig. 2. At the low concentrations of 0.2 and $0.5 \mathrm{wt} \%$, the scattering profiles $I(Q)$ are almost identical and independent of the concentration, suggesting that we are seeing an isolated micelle. At the high concentrations of 2 and 


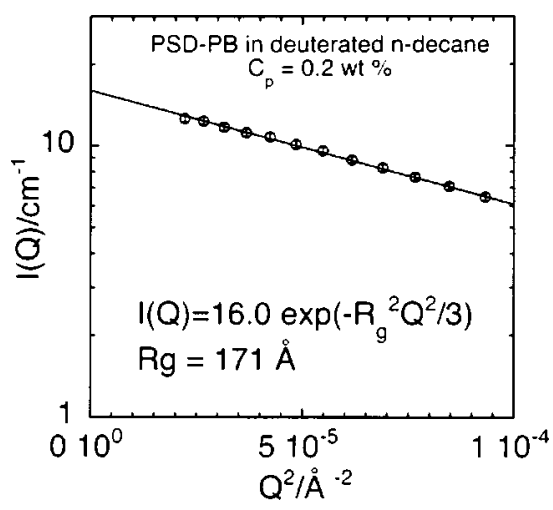

FIG. 3. Guinier plot of SANS intensity from $0.2 \mathrm{wt} \%$ PSD-PB diblock copolymer micelle in deuterated $n$-decane solution in a $Q$ range below $0.01 \AA^{-1}$.

20 wt $\%$, on the other hand, the intensity $I(Q)$ decreases in the low $Q$ range below about $0.01 \AA^{-1}$ with increasing concentration. This indicates structure factor effects that result from mutual repulsion of micelles. Careful inspection of the normalized intensities of the 0.2 and $0.5 \mathrm{wt} \%$ solutions in the inset-the $0.2 \mathrm{wt} \%$ solution has slightly higher intensity below about $0.007 \AA^{-1}$ —suggests that small interference effects still exist even in the $0.5 \mathrm{wt} \%$ solution. Therefore, in order to evaluate the form factor of an isolated micelle we analyzed the SANS data of the $0.2 \mathrm{wt} \%$ solution.

As a first approximation, we assume that the polymer micelle can be described by a hard-core-shell model which is schematically illustrated in Fig. 1. The scattering intensity $[I(Q)=\partial \Sigma / \partial \Omega]$ from this model is easily calculated and given by,

$$
\begin{aligned}
I(Q)=\frac{\partial \Sigma}{\partial \Omega}= & \frac{C_{w} \rho_{\mathrm{sol}} N_{A}}{M_{\mathrm{tot}} N_{\mathrm{agg}}}\left(\frac{4 \pi}{3}\right)^{2}\left[\phi_{S}\left(\rho_{b, B}-\rho_{b, \mathrm{sol}}\right)^{2}\right. \\
& \times\left\{R_{2}^{3} F\left(Q R_{2}\right)-R_{1}^{3} F\left(Q R_{1}\right)\right\} \\
& \left.+\phi_{c}\left(\rho_{b, \mathrm{SD}}-\rho_{b, \mathrm{sol}}\right) R_{1}^{3} F\left(Q R_{1}\right)\right]^{2},
\end{aligned}
$$

where $M_{\mathrm{tot}}$ and $\rho_{\mathrm{sol}}$ are the total molecular weight and the mass density of the solvent (deuterated $n$-decane), $\rho_{b, \text { sol }}, \rho_{b, B}$, and $\rho_{b, \mathrm{SD}}$ are the scattering length densities of solvent, $\mathrm{PB}$ block, and PSD block, $C_{w}$ and $N_{\text {agg }}$ are the weight concentration of polymers and the aggregation number of polymers in a micelle, and $\phi_{S}$ and $\phi_{C}$ are the volume fractions of PB in the shell and PSD in the core, respectively. $R_{2}$ and $R_{1}$ are the outer radii of the shell and core. $F(x)$ is the normalized scattering amplitude of a sphere

$$
F(x)=\frac{3[\sin (x)-x \cos (x)]}{x^{3}} .
$$

If we assume homogeneous densities within the shell and core, the volume fractions follow as

$$
\phi_{S}=\frac{M_{B} N_{\mathrm{agg}}}{\rho_{B} N_{A}(4 \pi / 3)\left[R_{2}^{3}-R_{1}^{3}\right]}, \quad \phi_{C}=\frac{M_{\mathrm{SD}} N_{\mathrm{agg}}}{\rho_{\mathrm{SD}} N_{A}(4 \pi / 3)\left[R_{1}^{3}\right]},
$$

where $M_{\mathrm{PB}}$ and $M_{\mathrm{PSD}}$ are the molecular weights of $\mathrm{PB}$ and PSD blocks, and $\rho_{B} \approx 0.9 \mathrm{~g} / \mathrm{cm}^{3}$ and $\rho_{\mathrm{SD}} \approx 1.12 \mathrm{~g} / \mathrm{cm}^{3}$ are the mass densities of PB block and PSD block, respectively. In this experiment, the scattering length density of the core

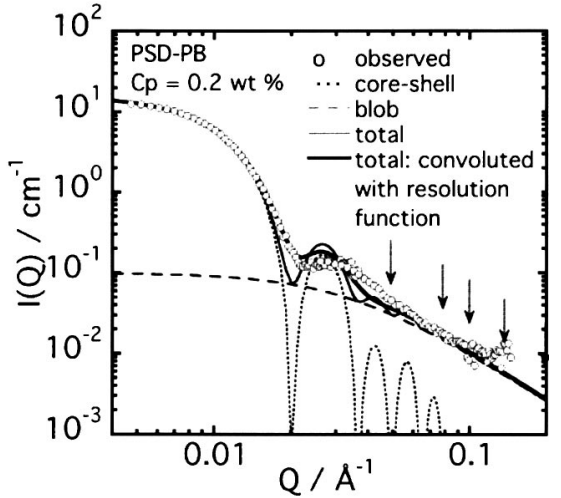

FIG. 4. Observed and calculated SANS intensities $I(Q)$ from 0.2 wt $\%$ PSD-PB diblock copolymer micelle in deuterated $n$-decane solution. $(O)$ : observed, $(\cdots)$ : core-shell scattering contribution, (- - -): blob scattering contribution, (-): sum of core-shell and blob scattering contributions without convoluting with resolution function of the SANS machine, and (-): sum of core-shell and blob scattering contributions after convoluting with resolution function of the SANS machine. The down arrows show the $Q$ values of NSE measurements.

consisting of PSD blocks is almost the same as that of the solvent (see Fig. 1), and the contrast factor of the core $\rho_{b, \mathrm{SD}}-\rho_{b, \mathrm{sol}}$ is extremely small. Hence, Eq. (1) can be approximated to

$$
\begin{aligned}
\frac{\partial \Sigma}{\partial \Omega}= & \frac{C_{W} \rho_{\mathrm{sol}} M_{B}^{2} N_{\mathrm{agg}}}{\rho_{B}^{2} M_{\mathrm{tot}} N_{A}}\left(\rho_{b, B}-\rho_{b, \mathrm{sol}}\right)^{2} \\
& \times\left[\frac{R_{2}^{3} F\left(Q R_{2}\right)-R_{1}^{3} F\left(Q R_{1}\right)}{R_{2}^{3}-R_{1}^{3}}\right]^{2},
\end{aligned}
$$

where the expression in brackets represents the normalized form factor $P(Q)$ of a hollow sphere and approaches 1 for $Q \rightarrow 0$.

For an isolated object, not knowing the form factor $P(Q)$, the scattering intensity can be described by the Guinier equation in a low $Q$ range below $1 / R_{g}$, where $R_{g}$ is a radius of gyration of the object. In the present experiment, it is given by

$$
\begin{aligned}
& \frac{\partial \Sigma}{\partial \Omega}=I(0) \exp \left(-\frac{R_{g}^{2} Q^{2}}{3}\right), \\
& I(0)=\frac{C_{W} \rho_{\mathrm{sol}} M_{B}^{2} N_{\mathrm{agg}}}{\rho_{B}^{2} M_{\mathrm{tot}} N_{A}}\left(\rho_{b, B}-\rho_{b, \mathrm{sol}}\right)^{2} .
\end{aligned}
$$

According to Eq. (3) we plotted the logarithm of the scattering intensity against $Q^{2}$ in the $Q$ range below $0.01 \AA^{-1}$ in Fig. 3. We have evaluated the prefactor $I(0)$ and radius of gyration $R_{g}$ from the straight line in the figure, as $16.0+ \pm 0.2 \mathrm{~cm}^{-1}$ and $171 \pm 2 \AA$, respectively. The aggregation number $N_{\mathrm{agg}}$ in Eq. (3) was calculated from $I(0)\left(=16.0 \mathrm{~cm}^{-1}\right)$ to be 78.7. From the aggregation number $N_{\text {agg, }}$, we have calculated the radius of the core $R_{1}$ to be $68 \AA$ under an assumption that the density of the core is the same as that in bulk PSD, i.e., $\phi_{C}=1$. The radius of gyration $R_{g}$ of an isolated micelle must be given as that of a hollow sphere because the scattering length density of the PSD core is almost the same as that of the solvent (deuterated $n$-decane). The radius of gyration of a hollow sphere is given by ${ }^{28}$ 


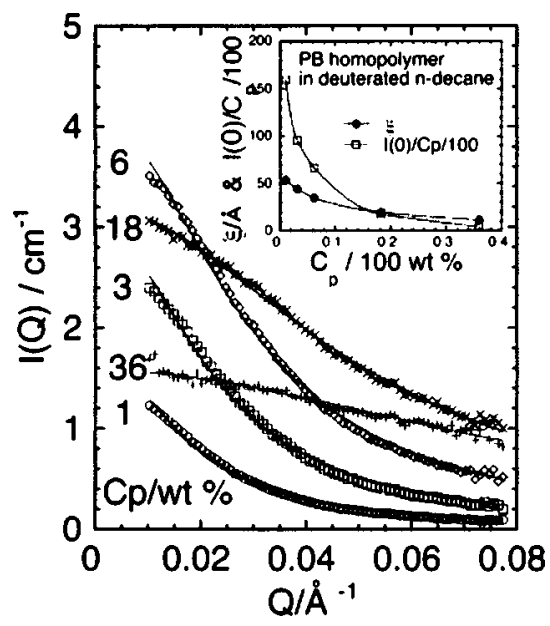

FIG. 5. SANS intensities of PB homopolymer in deuterated $n$-decane solution for various concentrations. The solid curves are the results of fits using Ornstein-Zernick equation $\left[I(Q)=I(0) /\left(1+Q^{2} \xi^{2}\right)\right]$. In the inset, evaluated $I(0)$ and $\xi$ are plotted as a function of polymer concentration.

$$
R_{g}^{2}=\frac{3\left(1-\alpha^{5}\right) R_{1}^{2}}{5\left(1-\alpha^{3}\right) \alpha^{2}}, \quad \alpha=R_{1} / R_{2}
$$

With the core radius $R_{1}(=68 \AA)$ we get the total radius of micelle $R_{2}=217 \AA$ through Eq. (4). Now we can calculate the scattering intensity from a dilute micelle solution in absolute units using the core-shell model Eq. (1) by inserting the parameters given above without further curve fitting. The calculated intensity is plotted in Fig. 4 as a dotted line, which agrees with the observed intensity in a low $Q$ range below about $0.015 \AA^{-1}$ while it decreases much faster than the observed one as $Q$ increases. This indicates that there are density fluctuations on shorter length scales within the micelle structure. The most plausible origin is chain fluctuations in the corona, which is the so-called blob scattering $I_{\text {blob }}(Q)$ that adds to the average shape micelle scattering $I(Q)$, i.e., $\partial \Sigma / \partial \Omega=I(Q)+I_{\text {blob }}(Q)$.

We have tried to estimate the scattering intensity due to the fluctuations experimentally assuming that the chain fluctuations in the corona may be compared to those in a PB solution with a concentration that corresponds to $\phi_{B}$. For this purpose, SANS measurements were carried out on deuterated $n$-decane solutions of PB homopolymer with $M_{w}=34000$ as a function of PB concentration. The observed SANS intensities are shown in Fig. 5 and fitted to the Ornstein-Zernike equation

$$
I(Q)=\frac{I(0)}{1+\xi^{2} Q^{2}},
$$

where $\xi$ and $I(0)$ are a correlation length and the intensity at $Q=0$. The results of fits show good agreements. From the fits we have evaluated the correlation length $\xi$ and $I(0)$ and plotted in the inset of Fig. 5 as a function of the polymer concentration. Using the structure parameters of the micelle $R_{1}, R_{2}$, and $N_{\text {agg }}$ evaluated above, the average concentration of PB blocks in the corona was calculated to be $8.6 \mathrm{wt} \%$ or $\phi_{B}=0.081$, respectively. At this concentration, the correlation length and the intensity $I(0)$ are $30.5 \AA$ and $4.48 \mathrm{~cm}^{-1}$, respectively, for the homopolymer solution. Putting these val-

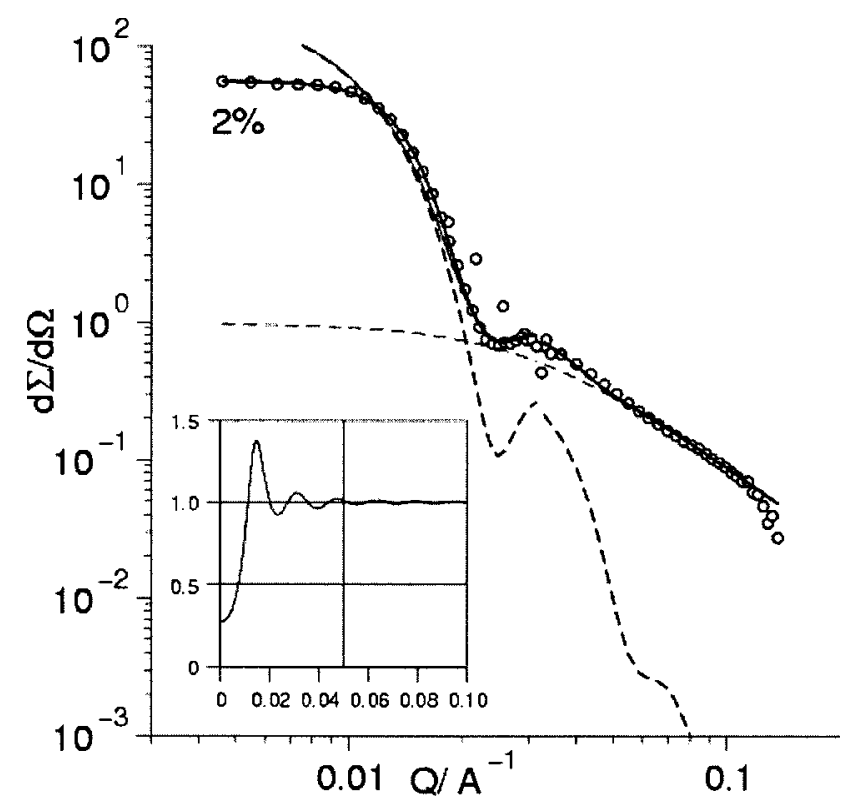

FIG. 6. Model fit of the $2 \%$ data with a core-shell model multiplied by a model structure factor. Thin dashed line: blob scattering contribution, thick dashed line: micelle contribution, and solid line: micelle contribution multiplied by the structure factor and added blob scattering. The insert shows the used model structure factor.

ues into Eq. (5) we have estimated the scattering intensity due to the concentration fluctuations in the corona. Finally, we obtained the total SANS intensity from the micelle as a sum of Eqs. (1) and (5). In Fig. 4, the scattering intensities due to the chain concentration fluctuations in the corona [Eq. (5)] and the total intensity are given by a long-dashed line and a thin solid line, respectively. The total computed scattering intensity was convoluted with the resolution function of the SANS machine, which is shown by a thick solid line. For this calculation of the SANS intensity we have estimated all the structural parameters from the direct experiments without model fitting. Nevertheless the agreement between the calculated and observed intensities is not bad. However, the calculated peak seems to appear at a $Q$ position $\left(=0.0266 \AA^{-1}\right)$ slightly smaller than the observed one $\left(=0.029 \AA^{-1}\right)$. This suggests that the real effective outer radius $R_{2}$ is slightly smaller than that evaluated from the radius of gyration $R_{g}$ within this model. Better agreement between the calculated and observed scattering intensities could be obtained by fitting the theoretical curve to the observed one by adjusting the structure parameters.

In a system of interacting spherical symmetric particles the scattering intensity $I(Q)$ can be described by the product of a single particle form factor $P(Q)$ and a structure factor $S(Q)$.

$$
I(Q)=P(Q) S(Q) .
$$

Note that the total scattering cross section is

$$
\frac{\partial \Sigma}{\partial \Omega}=P(Q) S(Q)+I_{\mathrm{blob}}(Q),
$$

since the fluctuation scattering is totally uncorrelated between different micelles such that any interference terms between neighboring particles average out to zero. 


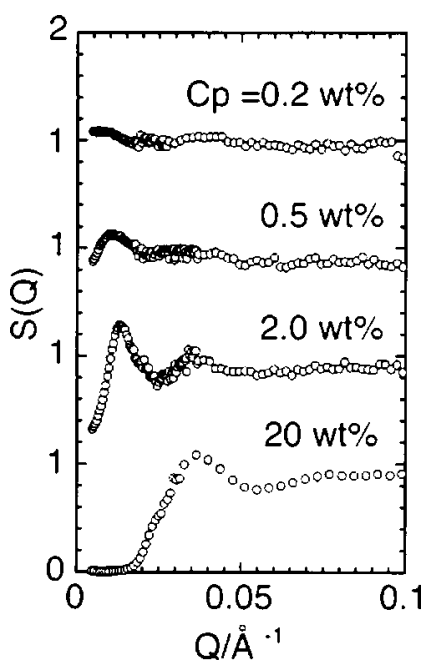

FIG. 7. Structure factor $S(Q)$ for the polymer concentration $C_{p}=0.2,0.5$, 2.0 , and $20 \mathrm{wt} \%$.

The $2 \%$ data have been fitted using the more flexible core-shell model where $S(Q)$ has been modeled by Sharma and Sharma ${ }^{29}$ and the blob scattering was added (Fig. 6). A perfect data fit can be obtained observing all mass conservation conditions imposed by the known molecular weights and concentrations. However, the aggregation number depends somewhat on the fit details [e.g., the $S(Q)$ model] within a range between 67 and 83. Also, the detailed form of the radial shell density may be varied somewhat within this approach. An ansatz of a radial density decay of the outer shell according to $\phi(r) \propto r^{-4 / 3}$ up to the outer shell radius is compared to a two-shell model with smeared boundaries. Both models fit the data in a way, as shown in Fig. 6. Here we arrive at the limits of the discrimination power between similar models of SANS without multiparameter contrast variation. The general features of a shell with decreasing density, with increasing radius, and an effective extension of about $250 \AA$ are corroborated by all model fits. Since mass conservation holds the outer radius obtained for constant radial density is lower $(217 \AA)$. Parameters for the model $S(Q)$ were hard-core radius $68 \AA$, potential-well width 250 (290) $\AA$, and potential-well height $1.9(1.1) k_{B} T$, where the values in parentheses relate to the smeared two-shell model.

Using the form factor $P(Q)$ evaluated above, we have extracted the structure factor $S(Q)$ for the micelle solutions and plotted in Fig. 7 at the concentration $C_{p}=0.2,0.5,2.0$, and $20 \mathrm{wt} \%$. It is obvious that the $S(Q)$ is unity at $C_{p}$ $=0.2 \mathrm{wt} \%$ because we observe an isolated micelle. In the $0.5,2.0$, and $20 \mathrm{wt} \%$ solutions, we observed a first correlation peak in $S(Q)$, which corresponds to the distance between the nearest neighboring micelles. It is hard to evaluate the peak position for the $0.5 \mathrm{wt} \%$ solution precisely due to the experimental error. At $2.0 \mathrm{wt} \%$, the peak position is about $0.012 \AA^{-1}$, giving the distance of $523 \AA$. The number density of micelles calculated from the aggregation number is $(1 / 636 \AA)^{3}$ inserting this (with $R_{2}=250$ A) into the SharmaSharma or Percus-Yevick form of the structure factor a first peak position of $Q=0.0122 \AA^{-1}$ results in perfect agreement with the experimental observation. The structure factor $S(Q)$ of the $20 \mathrm{wt} \%$ solution is not crystal-like, suggesting that the micelles are not well ordered. The distance between the nearest neighboring micelles is about $170 \AA$ for the 20 wt \% solution, which was calculated from the first peak position of $0.037 \AA^{-1}$. This is much smaller than twice the micelle radius $R_{2}(=217, \ldots, 270 \AA)$, suggesting that the micelles heavily interpenetrate and/or deform each other.

\section{B. Dynamics of the micellar corona}

In the next step, we investigated dynamics of the micelles in the 2.0 and $20 \mathrm{wt} \%$ solutions using a NSE technique at $Q=0.05,0.08,0.10,0.14,0.20$, and $0.24 \AA^{-1}$, which are indicated by the arrows in Fig. 4. In this $Q$ range the scattering intensity is dominated by the PB chain fluctuations in the corona. The scattering contribution from the average shape (dotted line in Fig. 4) is at least one order of magnitude smaller than the PB fluctuation scattering.

The observed normalized intermediate scattering functions $I(Q, t) / I(Q, 0)$ are shown for the 2 and $20 \mathrm{wt} \%$ solutions in Figs. 8(a) and 8(b), respectively, in a $Q$ range from 0.05 to $0.24 \AA^{-1}$. In this $Q$ range we mainly observed internal motions of polymer chains in the corona. The translational diffusion contributes to the relaxation of diffusing objects as multiplicative factor $\exp \left(-D Q^{2} t\right)$ to the internal dynamics. The diffusion coefficient may be estimated using the Einstein-Stokes value

$$
D=\frac{k_{B} T}{6 \pi \eta R_{H}} \approx 1.26 \AA^{2} / \mathrm{ns}
$$

with $\eta=0.73 \mathrm{cP}$ the estimated viscosity of deuterated $n$-decane at $40{ }^{\circ} \mathrm{C}$ and $R_{H}=250 \AA$ the estimated hydrodynamic radius of the micelles. Due to interaction as expressed in $S(Q)$ the effective diffusion constant may be modified, $D_{\text {eff }}(Q)=D H(Q) / S(Q)$ with $H(Q)$ the hydrodynamic factor. However, for the $2 \%$ sample the functions $S(Q)$ and $H(Q)$ in the $Q$ range of NSE experiments are close to 1 . This means that center-of-mass diffusion will lead to an extra drop by a factor 0.75 of the relaxation curve for $Q=0.1 \AA^{-1}$ and $t$ $=22 \mathrm{~ns}$. The measured relaxation drops to about 0.25 at this point which indicates that most of the relaxation observed at higher $Q$ is due to internal dynamics of the corona.

As shown in the structural studies, the micelles are not overlapped in the $2 \mathrm{wt} \%$ solution, but they are deeply interpenetrated in the $20 \mathrm{wt} \%$ solution. Thus the factorization of center-of-mass diffusion and internal dynamics may break down for the high-concentration sample. First we focus our attention on $I(Q, t) / I(Q, 0)$ of the 2 wt $\%$ solution or the isolated micelles. A far as we know, there are two reports on the dynamics of corona chains in isolated polymer micelles by NSE. ${ }^{19,20}$ In both of these studies it is claimed that the so-called breathing mode is observed, which is a collective motion of the corona chains driven by the osmotic compressibility and the chain entropic force. The theoretical prediction was given by de Gennes in $1986 .{ }^{18}$ According to the theory as well as the previous experimental studies, $I(Q, t) / I(Q, 0)$ of the breathing mode of corona chains is characterized by multiple component decay including very 

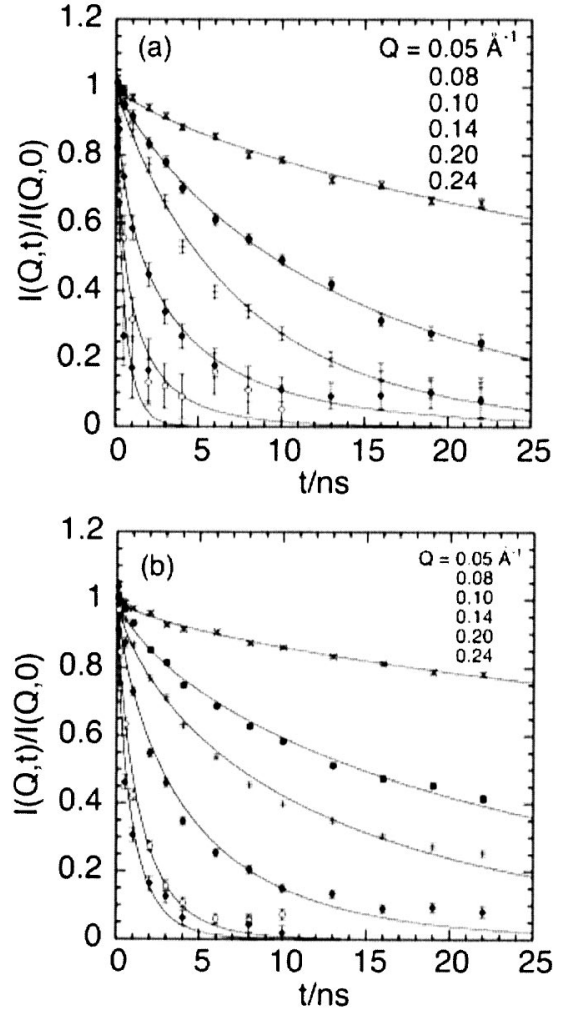

FIG. 8. Normalized intermediate scattering function $I(Q, t) / I(Q, 0)$ for PSD-PB diblock copolymer micelle in deuterated $n$-decane solutions. (a) $C_{p}=2.0 \mathrm{wt} \%$ and (b) $C_{p}=20 \mathrm{wt} \%$. The solid curves in the figures are guides to the eye by stretched exponential function fits.

fast and very slow decays from relaxation modes of the average segment concentration. In particular, the observed initial decays were considerably faster than the corresponding Zimm dynamics of the corona chains in a homopolymer solution. In the beginning of this study we also expected the breathing mode in this micelle system. However, the observed intermediate scattering function $I(Q, t) / I(Q, 0)$ of the $2 \mathrm{wt} \%$ solution of PSD-PB micelles is even slightly slower than that of the PB-homopolymer solution, as will be shown later, which renders it virtually impossible to identify any traces of a breathing mode. Which mode governs the motions of corona chains in this micelle? To shed light on this question NSE measurements on deuterated $n$-decane solutions of butadiene homopolymer with almost the same molecular weight $\left(M_{w}=36000, M_{w} / M_{n}=1.06\right)$ in the concentration range from 1 to $32 \mathrm{wt} \%$ were compared to those on the micellar dynamics. Apparently, the observed intermediate scattering functions $I(Q, t) / I(Q, 0)$ for the solutions are very similar to that of the isolated micelle solutions. In particular is the intermediate scattering function as obtained from a 8 wt \% PB-homopolymer solution nearly indistinguishable from that from the 2 wt $\%$ micellar solution, see Fig. 11. Here we note that the SANS analysis of the micelles gave an average polymer concentration in the corona of $8.6 \%$.

The standard model for the dynamics of polymer chains in dilute solutions is the Zimm model ${ }^{23}$ which contains the hydrodynamic coupling of chain segments and entropic forces between them as main ingredients. The intermediate
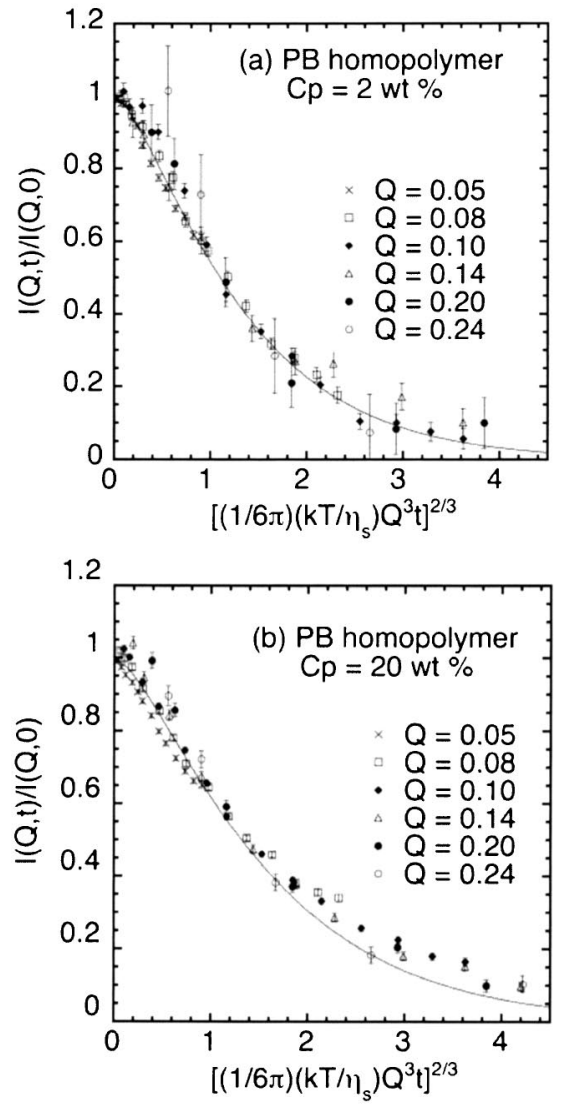

FIG. 9. Zimm scaling of normalized intermediate scattering function $I(Q, t) / I(Q, 0)$ for PB homopolymer in deuterated $n$-decane solutions. (a) $C_{p}=2 \mathrm{wt} \%$ and (b) $C_{p}=20 \mathrm{wt} \%$. The solid lines are Zimm master curve fitted to the observed data in the short-time region below $\left(W Q^{3} t\right)^{2 / 3}=1.5$.

scattering function of the Zimm model was theoretically calculated by de Gennes and Dubois-Violette ${ }^{30}$ and is given by

$$
\begin{aligned}
& \frac{I(\mathrm{Q}, t)}{I(\mathrm{Q}, 0)}=\int_{0}^{\infty} d u \exp \left\{-u-\left(\Gamma_{Z} t\right)^{2 / 3} h\left[\frac{u}{\left(\Gamma_{Z} t\right)^{2 / 3}}\right]\right\}, \\
& h(y)=\frac{4}{\pi} \int_{0}^{\infty} d x \frac{\cos x^{2}}{x^{3}}\left[1-\exp \left(-y^{-3 / 2} x^{3}\right)\right], \\
& \Gamma_{Z}=\frac{1}{6 \pi} \frac{k_{B} T}{\eta_{s}} Q^{3} .
\end{aligned}
$$

According to this calculation, the Zimm rate constant $\Gamma_{Z}$ is given by $(1 / 6 \pi)\left(k T / \eta_{s}\right) Q^{3}\left(=314 \mathrm{~ns}^{-1} \AA^{3} Q^{3}\right)$, where $\eta_{s}(=0.73 \mathrm{cP})$ is the viscosity of the solvent, and the intermediate scattering function $I(Q, t) / I(Q, 0)$ can be scaled by the so-called Zimm time $\left(\Gamma_{Z} t\right)^{2 / 3}$. Then, the observed $I(Q, t) / I(Q, 0)$ is plotted against the Zimm time $\left(W Q^{3} t\right)^{2 / 3}\left[W=(1 / 6 \pi)\left(k T / \eta_{s}\right)=314 \mathrm{~ns}^{-1} \AA^{3}\right]$ in Figs. 9(a) and 9 (b) for the 2 and $20 \mathrm{wt} \%$ solutions of PB homopolymer. Note that, e.g., at $Q=0.1 \mathrm{~A}^{-1}$ the estimated initial rate contribution due to center-of-mass diffusion is $0.0126 \mathrm{~ns}^{-1}$ whereas the Zimm dynamics contribution is computed as $0.314 \mathrm{~ns}^{-1}$ about 25 times the center-of-mass diffusion rate. As seen in Fig. 9(a), the Zimm scaling apparently works well for the $2 \mathrm{wt} \%$ PB-homopolymer solution and all the data points fall on the master curve, however, the effective sol- 

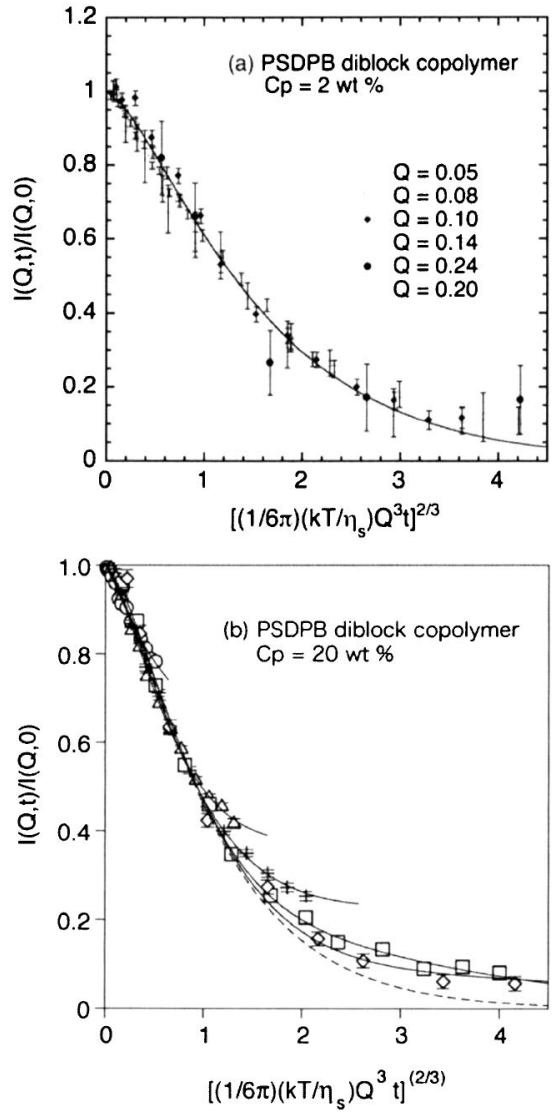

FIG. 10. Zimm scaling of normalized intermediate scattering function $I(Q, t) / I(Q, 0)$ for PSD-PB diblock copolymer micelle in deuterated $n$-decane solutions. (a) $C_{p}=2 \mathrm{wt} \%$. The solid line is Zimm model function fitted to the observed data in the short-time region below $\left(W Q^{3} t\right)^{2 / 3}=1.5$ with $\eta=1.74 \mathrm{cP}$. (b) $C_{p}=20 \mathrm{wt} \%$. The lines through the data points are guides to the eye only. At long times clear deviations from a single master curve are observed.

vent viscosity (fitted solid line) is $0.98 \mathrm{cP}$ rather than the expected $0.73 \mathrm{cP}$. In the more concentrated solutions $(16 \%$, $20 \%$, and $32 \%$ ), on the other hand, deviations from the Zimm scaling appear and deviations from the Zimm master curve are significant. Only the short-time behavior (initial slope) remains consistent with the Zimm expectation for the given solvent viscosity. This obvious deviation begins at a certain concentration between 4 and $8 \mathrm{wt} \%$, and is enhanced as the concentration increases. Comparing the decay curve of the $20 \mathrm{wt} \%$ solution with that of the $2 \mathrm{wt} \%$ (see Fig. 9), the overall motional slowing down in the $20 \mathrm{wt} \%$ solution is clearly observed. The slowing down and the deviation from the master curve must be predominantly caused by the increase of the effective viscosity with concentration (concentration effect) rather than by the entanglements since the molecular weight of the PB chains is slightly smaller than the critical entanglement molecular weight. ${ }^{31}$

For the $2 \mathrm{wt} \%$ PSD-PB micelle solution the intermediate scattering function $I(Q, t) / I(Q, 0)$ is plotted as a function of the Zimm time $\sim\left(W Q^{3} t\right)^{2 / 3}$ in Fig. 10(a). The Zimm scaling holds well within the available statistical accuracy for the isolated micelle solution, at least in the short-time region below $\left(W Q^{3} t\right)^{2 / 3}=3.5$, while there are no signs of a breathing mode. The question why it is not observed will be discussed

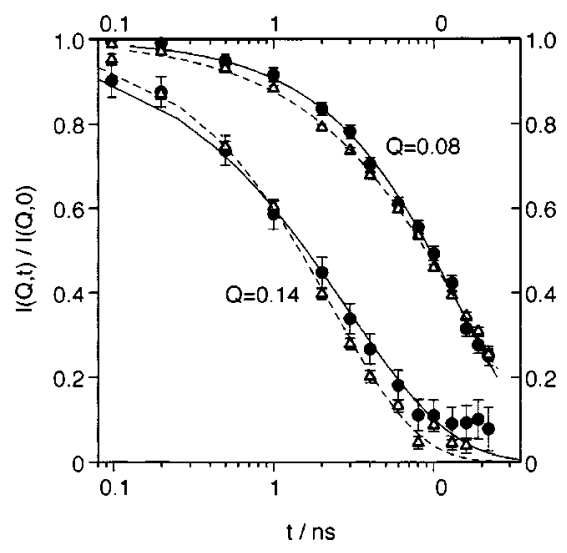

FIG. 11. Comparison of relaxation curves from the 2\% PSD-PB solution (solid circles) with those from a $8 \mathrm{wt} \% \mathrm{~PB}$ solution (open triangles) for $Q=0.08$ and $0.14 \mathrm{~A}^{-1}$. The solid and dashed lines are stretched exponential fits for the PSD-PB and PB data, respectively.

later. The master curve fitted to the observed data for the isolated micelle solution is shown in Fig. 10(a). The decay rate in the micelle solution is clearly slowed down compared with that in the homopolymer solution and the effective viscosity amounts to $1.3 \mathrm{cP}(1.2 \mathrm{cP})$ if a center-of-mass diffusion of $1.26 \mathrm{~A}^{2} / \mathrm{ns}$ is (not) included, resulting in a decay rate ratio of 0.80 . The scaling property of the decay curves from the micelle indicates that the brush dynamics is close to that of a polymer solution with Zimm dynamics. The effective chain concentration of $8.6 \mathrm{wt} \%$ in the corona as determined from the SANS results suggests a direct comparison with the $8 \mathrm{wt} \%$ homopolymer solution. Indeed the comparison reveals a better match between the $2 \mathrm{wt} \%$ micellar solution and the $8 \mathrm{wt} \% \mathrm{~PB}$-homopolymer solution than between the 2 wt $\%$ micellar solution and the 4 and $16 \mathrm{wt} \%$ homopolymer solutions. The best match between $I(Q, t) / I(Q, 0)$ of 2 wt $\%$ PSD-PB and 8 wt $\%$ PB is observed at $Q=0.08 \mathrm{~A}^{-1}$ (see Fig. 11). Still there are subtle differences in the shape of the curves which may be expressed in terms of a stretching exponents $\beta$ which are $(0.871 \pm 0.03,0.757 \pm 0.01)$ from fits to $\exp \left[-(t / \tau)^{\beta}\right]$ and $\tau=14.4 \pm 0.4 \mathrm{~ns}$ in both cases. In the relevant range (i.e., where $1>S(q, t) / S(q)>0.1)$ the Zimm model function matches a stretched exponential with $\beta$ $=0.85$ with a maximum error of 0.015 . Deviations from this $\beta$ value indicate and quantify deviations from a pure Zimmtype behavior.

The overall $Q$ dependence of the rate is determined by fitting the data for different $Q$ values-including data from individual zones on the detector to increase $Q$ resolution-to stretched exponential functions. The stretching exponent was fixed to $\beta=0.77$ which yields fair fits over the whole $Q$ range. Thereby Fig. 12 was obtained. It is clearly seen that over the whole $Q$ range $0.035<Q<0.2 \mathrm{~A}^{-1}$ a $Q^{3}$ dependence of the rate $1 / \tau$ is observed. The slope depends on the concentration of the homopolymer solutions and for the 8 wt $\%$ solution nearly matches the slope obtained for the $2 \%$ PSD-PB micellar system.

The $I(Q, t) / I(Q, 0)$ of the 20 wt \% micelle solution is also plotted as a function of the Zimm time $\left(W Q^{3} t\right)^{2 / 3}$ in Fig. $10(\mathrm{~b})$, showing that the scaling works in the short-time region below about $\left(W Q^{3} t\right)^{2 / 3}=1.0$ while it deviates by slowing 


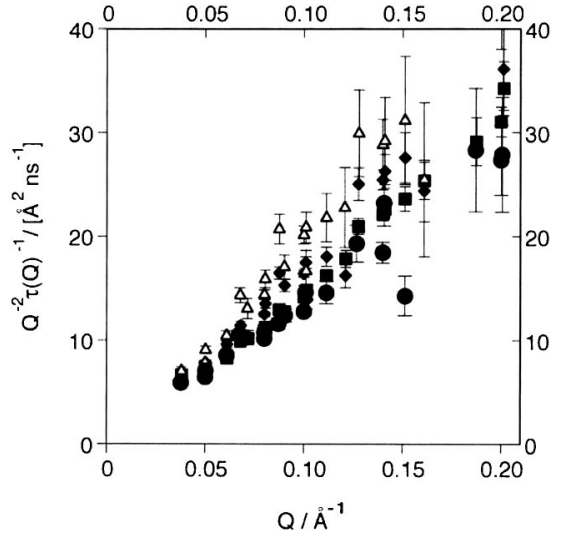

FIG. 12. Effective diffusion as function of $Q$ for the $2 \mathrm{wt} \%$ PSD-PB micellar solution (solid circles), the $1 \%$ homopolymer solution (triangles), the $4 \mathrm{wt} \%$ homopolymer solution (diamonds), and the $8 \mathrm{wt} \%$ solution (solid squares). The relaxation times are determined by stretched exponential fits with a fixed stretching exponent $\beta=0.77$.

down in the long-time region. The onset Zimm time when $I(Q, t) / I(Q, 0)$ deviates from the master curve depends on $Q$. The decay rate ratio compared to the dilute $(2 \mathrm{wt} \%) \mathrm{PB}$ homopolymer to that of the $20 \mathrm{wt} \%$ micelle solution estimated in the short-time region is 0.57 . Fitting the Zimm model to the data for the first $5, \ldots, 10 \mathrm{~ns}$ only yields an effective viscosity around $1.7 \mathrm{cP}$. The effective PB chain concentration is $14.7 \mathrm{wt} \%$, which was calculated by taking into account the overlap of the micelles.

For a comparison to the homopolymer solutions we fitted the data from the single detector zones with a stretched exponential $\exp \left[-(t / \tau)^{\beta}\right]$ with fixed $\beta=0.77$ which yields fair fits with a $\chi^{2}$ around 2 throughout. It is clearly seen in Fig. 13 that at low $Q$ the effective diffusion shown by the micellar coronae is much slower than that of the comparable solutions with 16 or $32 \mathrm{wt} \%$ homopolymer. We note that at the lowest $Q$ evaluated here the intermicelle structure factor $S(Q)$ as determined by SANS is already close to 1 and should not significantly influence the dynamics observed here. On a more local scale $\left(Q>0.1 \mathrm{~A}^{-1}\right)$ the behavior of the $20 \mathrm{wt} \%$ PSD-PB micellar solution approaches that of the 32 wt $\%$ homopolymer solution rather than that of the $16 \%$ solution, which is considerably faster.

The collective concentration fluctuations with longer wavelengths that lead to a plateau of $D_{\text {eff }}$ below about $Q$ $=0.07 \AA^{-1}$ in the most concentrated homopolymer solutions and below $Q=0.05 \AA^{-1}$ for the $16 \%$ solution are obviously suppressed in the micellar solution. This may be a consequence of the tethering of the chains to the micellar core that restricts their maximum displacements.

Finally, we would like to discuss why the breathing mode is not observed in the present micelle system while it was observed in other two systems. ${ }^{19,20}$ Characteristics of the micelles reported in Ref. 19 and this work are summarized in Table I where molecular weights of each block, aggregation number in a micelle, the grafting chain density on the surface of core $G$, and the average density (average volume fraction) of polymers in the corona $\phi_{s}$ are listed. The grafting chain density $G$ was calculated by

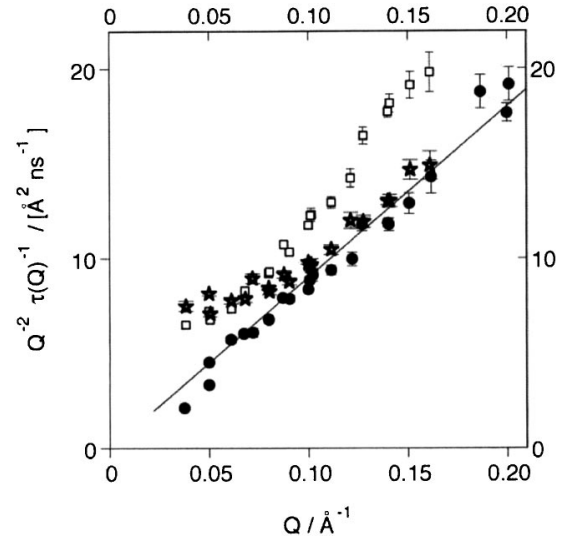

FIG. 13. Effective diffusion as function of $Q$ for the $20 \mathrm{wt} \%$ PSD-PB micellar solution (solid circles and line as guide to the eye), the 16 wt $\%$ homopolymer solution (squares), and the $32 \mathrm{wt} \%$ solution (stars). The relaxation times are determined by stretched exponential fits with a fixed stretching exponent $\beta=0.77$.

$$
G=\left(\frac{1}{36 \pi}\right)^{1 / 3}\left[\frac{N_{\mathrm{agg}} \rho_{\text {core }}^{2} N_{A}^{2}}{M_{\text {core }}}\right]^{1 / 3},
$$

where $M_{\text {core }}$ is the molecular weight of PSD chain in the core $\left(=M_{\mathrm{PSD}}\right)$ and $\rho_{\text {core }}$ is the density of the core. The breathing mode is a collective motion of many chains driven by the osmotic compressibility of the chain segments. It is therefore trivial that the grafting chain density on the core surface and the resulting average density in the corona play important roles. The latter plays an essential role although they are correlated. If the polymer density in the corona is low enough the breathing mode is not observed, but the selfmotion of chains (the Zimm mode) is observed in the available $Q$ range.

As Table I shows, the present micelle has only a slightly smaller density but a larger molecular weight of the "hairs" in the corona than those in Ref. 19. It is also closer to a starlike micelle than (in particular) the one investigated in Ref. 19 which tends towards a "crew cut" type. Visibility of the breathing mode requires that it has a faster dynamics than the Zimm modes. In a homopolymer solution of the corre-

TABLE I. Characteristics of two micelles used in studies of corona dynamics.

\begin{tabular}{lcc}
\hline \hline Ref. & Farago et al. $^{\mathrm{a}}$ & This work \\
\hline Solvent & $n$-decane- $d_{22}$ & $n$-decane- $d_{22}{ }^{\mathrm{b}}$ \\
Polymer & PSD-PI $^{\mathrm{c}}$ & PSD-PB $^{\mathrm{d}}$ \\
$M_{w}$ & $10000(\mathrm{PSD})$ & $11100(\mathrm{PSD})$ \\
& $7500(\mathrm{PI})$ & $23000(\mathrm{~PB})$ \\
$N_{\mathrm{agg}}$ & 120 & 78.7 \\
$G^{\mathrm{f}}\left(\right.$ chains $\left./ \mathrm{nm}^{2}\right)$ & 0.169 & 0.137 \\
Average volume fraction $\phi$ & 0.118 & $0.081^{\mathrm{g}}(0.052)^{\mathrm{h}}$ \\
\hline
\end{tabular}

\section{${ }^{\mathrm{a}}$ Reference 19.}

${ }^{\mathrm{b}}$ Deuterated $n$-decane.

${ }^{\mathrm{c}} \mathrm{PSD}$ : deuterated polystyrene, PI: polyisoprene.

${ }^{\mathrm{d}} \mathrm{PB}$ : Polybutadiene.

${ }^{\mathrm{e} A g g r e g a t i o n ~ n u m b e r . ~}$

${ }^{\mathrm{f}}$ Grafting chain density

${ }^{\mathrm{g}}$ For $R_{1}=68 \AA$ and $R_{2}=217 \AA$ (hard-core-shell model).

${ }^{\mathrm{h}}$ For $R_{1}=68 \AA$ and $R_{2}=250 \AA$ (flexible core-shell model). 
sponding regime is reached if $Q$ gets small enough to see the diffusion-type behavior $\sim Q^{2}$ of the rate rather than the $\sim Q^{3}$ Zimm-type rate dependence which takes over at higher $Q$. The higher the collective, osmotic-pressure driven diffusion is, the larger is the $Q$ value where it meets the Zimm relaxation rate. In the present case the corona has a density of $8.6 \%$ (or $\phi_{s}=0.081$ ), at this concentration the homopolymer solution is still not in the regime where the osmotic-pressure driven density fluctuations dominate and lead to a constant $D_{\text {eff }}$. As Fig. 13 shows this becomes visible only at $16 \%$ and $32 \%$ concentrations. The osmotic compressibility is the value that mainly determines this range of visibility of the collective diffusion-type motion. For semidilute and concentrated solutions it strongly deviates from the ideal-gas value depending on the second virial coefficient $A_{2}$, the relevant parameter being $\delta \pi / \delta c \sim f\left(A_{2} M_{w} c\right) .{ }^{32}$ Differences in $A_{2}$ of PB and Polyisoprene (PI) in decane could be the reason for the observed difference in the visibility of the breathing mode. Unfortunately $A_{2}$ values for both polymers in decane are currently unavailable, however, published values ${ }^{33}$ of the second virial coefficient of PB and PI in another hydrocarbon solvent, cyclohexane at $25^{\circ} \mathrm{C}$, differ by a factor of 10 $\left(A_{2}{ }^{1,4-\mathrm{PB}}=1.23 \times 10^{-3} \mathrm{~cm}^{3} \mathrm{~mol} \mathrm{~g}{ }^{-1} \times\{M /(\mathrm{g} / \mathrm{mol})\}^{-0.212} ; A_{2}{ }^{\mathrm{PI}}\right.$ $\left.=1.32 \times 10^{-2} \mathrm{~cm}^{3} \mathrm{~mol} \mathrm{~g}^{-1} \times\{M /(\mathrm{g} / \mathrm{mol})\}^{-0.232}\right)$. If a similar relation would hold in decane also the tenfold larger $A_{2}$ of PI could readily explain the observed difference of the breathing modes.

The complete lack of a diffusive regime in the $20 \mathrm{wt} \%$ micelle system still needs an explanation. Here the segment concentration is close to the $16 \%$ homopolymer solution. However, the local solution (Zimm)-type motion is slower (close to those in the $32 \%$ solution) and on the other hand their $Q$ dependence follows approximately a $Q^{3}$ law to the lowest values of the available range. One may speculate that the tethering of the corona polymers to their cores and the high density of chains from neighbor micelles or backfolded chains, which increase the average corona density from $8.6 \%$ to $16 \%$ also close to the tethering points, causes this observation. The constraints by tethering appear to decrease the displacement amplitude such that the osmotic-pressure driven density mode becomes undetectable.

In this study, we have investigated structure and dynamics of deuterated styrene-protonated butadiene diblock copolymer micelles in an isolated condition as well as in overlap condition. We analyzed the scattering intensity from an isolated micelle using a simple hard-core-shell model without curve fitting, and found that it cannot reproduce the observed data without taking into account the chain fluctuations in the corona (blob scattering). The blob contribution was experimentally evaluated by measuring the homopolymer solutions as a function of the concentration, and it was found that the sum of the hard-core-shell model and the blob scattering well reproduce the observed data. In the dynamic studies of the PB chains in the corona by neutron spin echo, the breathing mode was not observed due to slightly too low effective segment concentration $A_{2} M_{w} c$ in the corona. The motion is well described by the Zimm mode for the 2.0 and $20 \mathrm{wt} \%$ solutions in a short-time region while it deviates from the Zimm mode for the $20 \%$ solution in the long-time region. The onset time of the deviation depends on $Q$. The Zimm decay rate $\Gamma_{Z}$ in the micelle solution was slowed down compared with that in the homopolymer solution. This slowing down has been assigned to the effective high concentration in the corona. The differences in $\Gamma_{Z}$ between concentrated solutions and the $20 \%$ micellar solution are attributed to end-tethering effect of the PB chains on the core surface.

${ }^{1}$ S. Krause, J. Phys. Chem. 68, 1948 (1964).

${ }^{2}$ A. Halperin, M. Tirrell, and T. P. Lodge, Adv. Polym. Sci. 100, 31 (1992).

${ }^{3}$ Z. Turar and P. Kratochvil, in Surface and Colloid Science, edited by E.

Matijevic (Plenum, New York, 1993), p.1.

${ }^{4} \mathrm{~B}$. Chu, Langmuir 11, 414 (1995).

${ }^{5}$ B. Chu, Laser Light Scattering (Academic, Boston, 1991).

${ }^{6}$ B. J. Berne and R. Pecora, Dynamic Light Scattering (Wiley, New York, 1976).

${ }^{7}$ A. Guinier and G. Fournet, Small-Angle Scattering of X-Rays (Wiley, New York, 1955).

${ }^{8}$ O. Glatter, in Small Angle X-Ray Scattering, edited by O. Glatter and O. Kratky (Academic, London, 1982), p. 128.

${ }^{9}$ J. S. Higgins and H. C. Benoit, Polymers and Neutron Scattering (Clarendon, Oxford, 1994).

${ }^{10}$ T. Kotaka, T. Tanaka, and H. Inagaki, Polym. J. (Tokyo, Jpn.) 3, 327 (1972).

${ }^{11}$ T. Tanaka, T. Kotaka, and H. Inagaki, Polym. J. (Tokyo, Jpn.) 3, 338 (1972).

${ }^{12}$ C. Price, J. D. C. McAdam, T. P. Lally, and D. Woods, Polymer 15, 228 (1974).

${ }^{13}$ M. Nakano, M. Deguchi, K. Matsuomoto, H. Matsuoka, and H. Yamaoka, Macromolecules 32, 7437 (1999).

${ }^{14} \mathrm{P}$. Alexandridis and L. Yang, Macromolecules 33, 5574 (2000).

${ }^{15}$ Y. N. Lin and P. Alexandridis, J. Phys. Chem. B 106, 12124 (2002).

${ }^{16}$ H. Matsuoka, Y. Yamamoto, M. Nakano et al., Langmuir 16, 9177 (2000).

${ }^{17}$ K. Gohr, W. Schartl, W. Willner, and W. Pyckhout-Hintzen, Macromolecules 35, 9110 (2002).

${ }^{18}$ P. G. de Gennes, C. R. Acad. Sci., Ser. II: Mec., Phys., Chim., Sci. Terre Univers 302, 765 (1986).

${ }^{19}$ B. Farago, M. Monkenbusch, D. Richter, J. S. Huang, L. J. Fetters, and A. P. Gast, Phys. Rev. Lett. 71, 1015 (1993).

${ }^{20}$ T. Kanaya, H. Watanabe, Y. Matsushita, T. Takeda, H. Seto, M. Nagao, Y. Fujii, and K. Kaji, J. Phys. Chem. Solids 60, 1367 (1999).

${ }^{21}$ M. Monkenbusch, D. Schneiders, D. Richter, B. Farago, L. Fetters, and J. Huang, Physica B 213/214, 707 (1995).

${ }^{22}$ In Neutron Spin Echo, edited by F. Mezei (Springer, Berlin, 1979).

${ }^{23}$ B. Ewen and D. Richter, Adv. Polym. Sci. 134, 1 (1997).

${ }^{24}$ H. Watanabe, T. Kanaya, and Y. Takahashi, Macromolecules 34, 662 (2001).

${ }^{25}$ D. Schwahn, G. Meier, and T. Springer, J. Appl. Crystallogr. 24, 568 (1991).

${ }^{26}$ Y. Ito, M. Imai, and S. Takahashi, Physica B 213/214, 889 (1995).

${ }^{27}$ M. Monkenbusch, R. Schaetzler, and D. Richter, Nucl. Instrum. Methods Phys. Res. A 399, 301 (1997).

${ }^{28}$ Y. Hiragi and S. Ihara, Acta Crystallogr., Sect. A: Cryst. Phys., Diffr., Theor. Gen. Crystallogr. 37, 378 (1981).

${ }^{29}$ R. V. Sharma and K. C. Sharma, Physica A 89, 213 (1977).

${ }^{30}$ P. G. de Gennes and E. Dubois-Violette, Physics (Long Island City, N.Y.) 3, 181 (1967).

${ }^{31}$ In Polymer Data Handbook, edited by J. E. Mark (Oxford University Press, Oxford, 1999).

${ }^{32}$ C. I. D. Bica, W. Burchard, and R. Stadler, Eur. Polym. J. 33, 1759(1997).

${ }^{33}$ L. J. Fetters, N. Hadjichristides, J. S. Lindner, and J. W. Mays, J. Phys. Chem. Ref. Data 29, 619 (1994). 